\title{
NOETHERIAN SUBRINGS OF POWER SERIES RINGS
}

\author{
DAQING WAN
}

(Communicated by Wolmer Vasconcelos)

\begin{abstract}
Let $R$ be a commutative noetherian ring with unit. It is shown that certain subrings contained between the polynomial ring $R[X]$ and the power series ring $R[X][[Y]]$ are also noetherian. These subrings naturally arise from studying $p$-adic analytic variation of zeta functions over finite fields.
\end{abstract}

\section{INTRODUCTION}

Let $R$ be a commutative noetherian ring with unit, and let $R[X]=$ $R\left[X_{1}, \cdots, X_{n}\right]$ be the polynomial ring of $n$-variables over $R$. Hilbert's basis theorem asserts that $R[X]$ is noetherian. Let $R[X][[Y]]=R[X]\left[\left[Y_{1}, \cdots, Y_{m}\right]\right]$ be the formal power series ring over the noetherian ring $R[X]$. It is well known that $R[X][[Y]]$ is also noetherian. Motivated by Dwork's $p$-adic theory, it is of interest to study certain noetherian subrings which are contained between $R[X]$ and $R[X][[Y]]$.

Let $S_{k}$ be the set of polynomials in $R[X, Y]$ which are homogeneous in $Y$ of degree $k$. An element of the power series ring $R[X][[Y]]$ can be written uniquely in the form

$$
f=\sum_{k=0}^{\infty} f_{k}(X, Y), \quad f_{k}(X, Y) \in S_{k} .
$$

In this expansion, there is no restriction on the size of the degree $d_{k}$ of $f_{k}(X, Y)$ viewed as a polynomial in $X$. In Dwork's $p$-adic theory, one would want to study certain $f$ such that the degree function $d_{k}$ is bounded by another function in $k$. For this purpose, let $\lambda(x)$ be a non-negative increasing function defined over the non-negative real numbers. Define a subset of $R[X][[Y]]$ as follows:

$$
R[X ; Y, \lambda]=\left\{f=\sum_{k=0}^{\infty} f_{k} \mid f_{k} \in S_{k}, \operatorname{deg}_{X}\left(f_{k}\right) \leq C_{f}(\lambda(k)+1)\right\},
$$

where $C_{f}$ is a constant depending only on $f$. Equivalently, if $\lambda(x)$ is not

Received by the editors October 18, 1993.

1991 Mathematics Subject Classification. Primary 13E05, 13F25, 13 J05.

Partially supported by the National Science Foundation. 
identically zero, then

$$
R[X ; Y, \lambda]=\left\{f=\sum_{k=0}^{\infty} f_{k} \mid f_{k} \in S_{k}, \operatorname{deg}_{X}\left(f_{k}\right) \leq C_{f} \lambda(k) \text { for } k \gg 0\right\} .
$$

It is clear that $R[X ; Y, \lambda]$ is an $R[X]$-module containing $R[X]$. Since $\lambda(x)$ is increasing, we have the trivial inequality

$$
\lambda(x)+\lambda(y) \leq 2 \lambda(x+y) .
$$

This inequality immediately implies that the usual multiplication operation is closed for the set $R[X ; Y, \lambda]$. Thus, we have

Lemma 1.1. Let $\lambda(x)$ be a non-negative increasing function on the non-negative real numbers $\mathbb{R}_{\geq 0}$. Then $R[X ; Y, \lambda]$ is a ring, and hence an $R[X]$-algebra.

From the definition, one sees that $R[X ; Y, \lambda]$ depends only on the values of $\lambda(x)$ for large $x$. Furthermore, for any positive constant $c$, we have $R[X ; Y, c \lambda]=R[X ; Y, \lambda]$. If we take $\lambda(x)=\infty$, then $R[X ; Y, \lambda]$ is the full ring $R[X][[Y]]$. If we take $\lambda(x)$ to be a positive constant, then $R[X ; Y, \lambda]$ is the ring $R[[Y]][X]$. If we take $\lambda(x)=x$, then $R[X ; Y, \lambda]$ is the so-called overconvergent subring of $R[X][[Y]]$ as studied in the theory of Dwork-MonskyWashnitzer [4]. In all of these cases, the ring $R[X ; Y, \lambda]$ is known to be noetherian. It is then natural to ask

Question 1.2. Is the ring $R[X ; Y, \lambda]$ always noetherian?

It was first proved by Fulton [2] that the above question has a positive answer for certain $\lambda(x)$, including the power function $\lambda(x)=x^{r}$, where $r \geq 1$ is a real number. In studying $p$-adic analytic variations of zeta functions and $L$ functions (Dwork-Sperber [1] and Wan [5]), one is led to analytic functions with logarithmic decay or exponential growth. In the notation here, this means that we need to study elements in the ring $R[X ; Y, \lambda]$ with $\lambda(x)=e^{r x}$ for $r>0$.

Our purpose in this note is to show that $R[X ; Y, \lambda]$ is noetherian for a general class of functions $\lambda(x)$ (including the exponential function $e^{r x}$ ). We have

Theorem 1.3. Assume that there is another positive valued function $\mu(x) \geq 1$ such that for all sufficiently large $x$ and $y$,

$$
\lambda(x)+\lambda(y) \leq \lambda(x+y) \leq \lambda(x) \mu(y) .
$$

Then $R[X ; Y, \lambda]$ is noetherian.

One checks that the exponential function $\lambda(x)=e^{r x}(r>0)$ satisfies inequality (1.2) for large $x$ and $y$ (for example, one can take $\mu(y)=e^{r y}$ ). More generally, the growth function $\lambda(x)=e^{r x^{s}}(r>0$ and $0<s \leq 1)$ satisfies inequality (1.2). We conclude that $R[X ; Y, \lambda]$ is noetherian for such $\lambda(x)$. Inequality (1.2) shows that $\lambda(x)$ grows at least as fast as linear functions and grows at most as fast as exponential functions. Thus, for the slower growing function $\lambda(x)=x^{r} \quad(0<r<1)$ and the faster growing function $\lambda(x)=e^{r x^{s}}$ $(r>0$ and $s>1$ ), we do not know whether $R[X ; Y, \lambda]$ is noetherian. In fact, our feeling in these cases is somewhat negative. 
An alternative description of the ring $R[X ; Y, \lambda]$ is as follows: Let $\lambda^{-1}(x)$ be the inverse function of $\lambda(x)$ (assume that the inverse function exists). Let $R_{d}$ be the subset of $R[X][[Y]]$ which are homogeneous in $X$ of degree $d$. The elements in $R_{d}$ are not necessarily polynomials. For $f_{d} \in R_{d}$, define ord $Y\left(f_{d}\right)$ to be the largest integer $k$ such that $f_{d} \in Y^{k} R[X][[Y]]$, where $Y$ is viewed as the ideal generated by the $Y_{i}$. Then

$$
R[X ; Y, \lambda]=\left\{f=\sum_{k=0}^{\infty} f_{d} \mid f_{d} \in R_{d}, \operatorname{ord}_{Y}\left(f_{d}\right) \geq \lambda^{-1}\left(C_{f} d\right) \text { for } d \gg 0\right\} .
$$

\section{Proof of Theorem 1.3}

We may assume that $\lambda(x)$ is unbounded and $\mu(x) \geq 1$ for all $x$. If $\lambda(x)$ satisfies (1.2) only for large $x$ and $y$, then we can replace $\mu(y)$ by a larger function and redefine $\lambda(x)$ for small $x$ to be a suitable linear function so that $\lambda(x)$ satisfies (1.2) for all $x \geq 0, y \geq 0$ and furthermore $\lambda(0)=0$ and $\lambda(x)>0$ for $x>0$. We can then normalize $\lambda(x)$ (multiply $\lambda(x)$ by a suitable constant) so that $\lambda(x) \geq 1$ for $x \geq 1$. As we noted in the introduction, this will not change the ring $R[X ; Y, \lambda]$. Our proof attempts to follow the proof as given by Fulton [2] and keep careful track of the growth conditions. Instead of using only graded rings, we also use bigraded rings. Thus, our proof combines the lowest degree argument for the case of power series rings and the highest degree argument for the case of polynomial rings (see Lang [3]).

Let $S_{d, k}$ be the polynomials in $R[X, Y]$ which are homogeneous in $X$ of degree $d$ and homogeneous in $Y$ of degree $k$. One sees that $S_{d, k}$ is a free $R$-module with the natural base of monomials in $X$ and $Y$. Refining (1.1), each $f \in R[X][[Y]]$ has a unique representation

$$
f=\sum_{d, k=0}^{\infty} f_{d, k}, \quad f_{d, k} \in S_{d, k}
$$

Then

$$
R[X ; Y, \lambda]=\left\{f=\sum_{d, k=0}^{\infty} f_{d, k} \mid f_{d, k} \in S_{d, k}, f_{d, k}=0 \text { if } d>C_{f}(\lambda(k)+1)\right\} .
$$

Recall the defining property for a ring to be noetherian: every ideal of the ring is finitely generated or every ascending chain of ideals stops. Let $\lambda(x)$ be a non-negative increasing function satisfying inequality (1.2) and the above normalization. Let $J$ be an ideal of the ring $R[X ; Y, \lambda]$. We need to prove that $J$ is finitely generated.

For integers $d, k \geq 0$, let

$$
\begin{aligned}
M_{d, k}= & \left\{f_{d, k} \in S_{d, k}:\right. \\
& \text { there exists an element } \left.\left(\sum_{u=0}^{d} f_{u, k}+\sum_{u \geq 0, v \geq k+1}^{\infty} f_{u, v}\right) \in J\right\},
\end{aligned}
$$

where $f_{u, v} \in S_{u, v}$. Note that $\sum_{u=0}^{d} f_{u, k}$ is the lowest degree part of $f \in J$ in terms of $Y$ and $f_{d, k}$ is the highest degree part of $\sum_{u=0}^{d} f_{u, k}$ in terms of 
$X$. One checks that $S_{u, v} M_{d, k} \subset M_{d+u, k+v}$. The polynomial ring $R[X, Y]$ is a bi-graded ring with respect to $X$ and $Y$. The bi-homogeneous ideal $A$ of the noetherian ring $R[X, Y]$ generated by all bi-graded pieces $M_{d, k}$ is finitely generated. Thus, there are large integers $N_{1}$ and $N_{2}$ such that

$$
A=\sum_{i=0}^{N_{1}} \sum_{j=0}^{N_{2}} R[X, Y] M_{i, j} .
$$

Comparing the bi-homogeous parts, we deduce that for $u \geq N_{1}, v \geq N_{2}$,

$$
A_{u, v}=\sum_{i=0}^{N_{1}} \sum_{j=0}^{N_{2}} S_{u-i, v-j} M_{i, j} .
$$

Now,

$$
S_{u-i, v-j} M_{i, j}=S_{u-N_{1}, v-N_{2}} S_{N_{1}-i, N_{2}-j} M_{i, j} \subseteq S_{u-N_{1}, v-N_{2}} M_{N_{1}, N_{2}} .
$$

Thus,

We deduce that

$$
A_{u, v} \subseteq S_{u-N_{1}, v-N_{2}} M_{N_{1}, N_{2}} \subseteq M_{u, v} \subseteq A_{u, v} .
$$

$$
M_{u, v}=A_{u, v}=S_{u-N_{1}, v-N_{2}} M_{N_{1}, N_{2}}, \quad u \geq N_{1}, v \geq N_{2} .
$$

For each fixed integer $0 \leq d<N_{1}$, the $R[Y]$-module generated by the graded pieces $M_{d, k}(k \geq 0)$ is also noetherian (this module is a submodule of the noetherian $R[Y]$-module $\left.S_{d, 0} R[Y]\right)$. Thus, we can take a large integer $N_{2}(d)$ such that

$$
M_{d, k}=S_{0, k-N_{2}(d)} M_{d, N_{2}(d)}, \quad k \geq N_{2}(d) .
$$

Let $N=\max _{0 \leq d<N_{1}}\left(N_{2}, N_{2}(d)\right)$. Since $S_{u, v} S_{i, j}=S_{u+i, v+j}=S_{1,0}^{u+i} S_{0,1}^{v+j}$, it follows from (2.1) and (2.2) that

$$
\begin{aligned}
& M_{u, v}=S_{u-N_{1}, v-N} M_{N_{1}, N}, \quad u \geq N_{1}, \quad v \geq N . \\
& M_{d, k}=S_{0, k-N} M_{d, N}, \quad 0 \leq d<N_{1}, \quad k \geq N .
\end{aligned}
$$

For each fixed $0 \leq d \leq N_{1}$ (including $d=N_{1}$ ), choose a finite number of elements of the following form from $J$ indexed by $i$ :

$$
\begin{aligned}
Q(d, i) & =Q_{N}(d, i)+\sum_{k \geq N+1} Q_{k}(d, i), \\
& =\sum_{u=0}^{d} Q_{u, N}(d, i)+\sum_{k \geq N+1} Q_{k}(d, i)
\end{aligned}
$$

where $Q_{k}(d, i) \in S_{k}$, such that the leading terms $Q_{d, N}(d, i)$ (lowest degree in $Y$ and then highest degree in $X$ ) generate $M_{d, N}$ as $R$-module.

We now turn to the single grading defined with respect to $Y$ and describe similar properties. Recall that $S_{k}$ is the set of polynomials in $R[X, Y]$ which are homogenous in $Y$ of degree $k$ (the degree of $X$ is not counted). Let

$$
M_{k}=\left\{f_{k} \mid\left(f_{k}+\sum_{v \geq k+1}^{\infty} f_{v}\right) \in J, \quad f_{v} \in S_{v}\right\} .
$$


This is an $R[X]$-module. The homogeneous ideal of the noetherian ring $R[X, Y]$ generated by all the graded pieces $M_{k}$ is finitely generated (the grading is now only with respect to $Y$ ). Thus, we may assume that our integer $N$ is so large that

$$
M_{k}=S_{k-N} M_{N}, \quad k \geq N .
$$

For each fixed integer $0 \leq k<N$, we choose a finite number of elements of the following form from $\bar{J}$ indexed by $j$ :

$$
P(k, j)=\sum_{v=k}^{\infty} P_{v}(k, j) \in J, \quad P_{v}(k, j) \in S_{v},
$$

such that the leading terms $P_{k}(k, j)$ (the lowest degree term in $Y$ ) generate $M_{k}$ as $R[X]$-module.

We claim that if $k \geq N$ and $f_{k}=\sum_{u=0}^{r} f_{u, k} \in M_{k}$, then there are finitely many polynomials $a(d, i)$ indexed by $d$ and $i$ such that

$f_{k}=\sum_{d, i} a(d, i) Q_{N}(d, i), \quad a(d, i) \in S_{k-N}, \operatorname{deg}_{X}(a(d, i)) \leq \operatorname{deg}_{X}\left(f_{k}\right)=r$.

To prove the claim, we first assume that $r \geq N_{1}$. By (2.3), there are polynomials $a(N, i) \in S_{k-N}$ such that

$$
f_{r, k}=\sum_{i} a(N, i) Q_{N_{1}, N}\left(N_{1}, i\right), \quad \operatorname{deg}_{X}(a(N, i)) \leq r-N_{1} .
$$

Since $Q_{N_{1}, N}\left(N_{1}, i\right)$ (resp. $f_{r, k}$ ) is the highest degree part (in the variables $X$ ) of the polynomial $Q_{N}\left(N_{1}, i\right) \in M_{N}$ (resp. $f_{k} \in M_{k}$ ), it follows from (2.7) that the polynomial $f_{k}-\sum_{i} a(N, i) Q_{N}(N, i) \in M_{k}$ has its degree in $X$ smaller than $r$. Repeating this procedure, we may assume that $r<N_{1}$. Applying the same method and using the $Q_{N}(d, i)$ for $d<N_{1}$, we conclude that the claim is true.

We now prove that $J$ is generated by the finitely many elements $Q(d, i)$ and $P(k, j)$ in $J$. Let $G \in J$. By subtracting an $R[X]$-linear combination of the $P(k, j)$, we may assume that $G=\sum_{k=N}^{\infty} G_{k}$, where $G_{k} \in S_{k}$. We need to find finitely many elements $h(d, i)=\sum_{k=0}^{\infty} h_{k}(d, i)\left(h_{k}(d, i) \in S_{k}\right)$ and a constant $C$ such that $G(X)=\sum_{d, i} h(d, i) Q(d, i)$ and $\operatorname{deg}_{X}\left(h_{k}(d, i)\right) \leq C(\lambda(k)+1)$ for all $k, i, d$.

By equation (2.6), we can choose polynomials $h_{0}(d, i) \in S_{0}$ such that

$$
G_{N}=\sum_{i} h_{0}(d, i) Q_{N}(d, i)
$$

Take a constant $C$ so large that $\operatorname{deg}_{X}\left(h_{0}(d, i)\right) \leq C$ for all $d, i$, and

$$
\operatorname{deg}_{X}\left(Q_{k}(d, i)\right) \leq \frac{C}{2 \mu(N)}(\lambda(k)+1), \quad \operatorname{deg}_{X}\left(G_{k}\right) \leq \frac{C}{\mu(N)}(\lambda(k)+1)
$$

for all $d, k, i$.

Let $k \geq 1$. Suppose that the $h_{v}(d, i) \in S_{v}$ have been found satisfying the inequality $\operatorname{deg}_{X}\left(h_{v}(d, i)\right) \leq C(\lambda(v)+1)$ for all $v \leq k-1$ and

$$
G_{N+u}=\sum_{d, i} \sum_{v=0}^{u} h_{v}(d, i) Q_{N+u-v}(d, i)
$$


for all $u \leq k-1$. By equation (2.6), we can then choose $h_{k}(d, i) \in S_{k}$ so that

$$
G_{N+k}-\sum_{d, i} \sum_{v=0}^{k-1} h_{v}(d, i) Q_{N+k-v}(d, i)=\sum_{d, i} h_{k}(d, i) Q_{N}(d, i) .
$$

Furthermore, by (2.6) the degree in $X$ of $h_{k}(d, i)$ is bounded by

$$
\begin{aligned}
\max & \left(\operatorname{deg}_{X} G_{N+k}, \max _{0 \leq v \leq k-1, d, i}\left\{\operatorname{deg}_{X}\left(h_{v}(d, i)\right)+\operatorname{deg}_{X}\left(Q_{N+k-v}(d, i)\right)\right\}\right) \\
& \leq \max \left(\frac{C}{\mu(N)}(\lambda(N+k)+1),\right. \\
& \left.\quad \max _{0 \leq v \leq k-1}\left\{C(\lambda(v)+1)+\frac{C}{2 \mu(N)}(\lambda(N+k-v)+1)\right\}\right) \\
& \leq \max \left(C(\lambda(k)+1), \max _{0 \leq v \leq k-1}\left\{C(\lambda(v)+1)+\frac{C}{2}(\lambda(k-v)+1)\right\}\right) \\
& \leq \max \left(C(\lambda(k)+1), \max _{0 \leq v \leq k-1}\{C(\lambda(v)+1)+C \lambda(k-v)\}\right) \\
& \leq C(\lambda(k)+1),
\end{aligned}
$$

where in the second inequality we used the conditions $\lambda(N+k) \leq \lambda(k) \mu(N)$ and $\mu(N) \geq 1$. The third inequality follows from our normalization that $\lambda(k-v) \geq$ 1 for all $k-v \geq 1$. By induction, we can then find the required $h(d, i)$. Thus, $J$ is a finitely generated ideal. The theorem is proved.

\section{ACKNOWLEDGMENT}

I would like to thank William Fulton for kindly explaining a key part of his proof in [2] and the referee for helpful comments.

\section{REFERENCES}

1. Dwork and S. Sperber, Logarithmic decay and overconvergence of the unit root and associated zeta function, Ann. Sci. École Norm. Sup. (4) 24 (1991), 575-604.

2. W. Fulton, A note on weakly complete algebras, Bull. Amer. Math. Soc. 75 (1969), 591-593.

3. S. Lang, Algebra, Addison-Wesley, Reading, MA, 1971, pp. 144-147.

4. P. Monsky and G. Washnitzer, Formal cohomology I, Ann. of Math. (2) 88 (1968), 181-217.

5. D. Wan, Meromorphic continuation of L-functions of p-adic representations, preprint.

Department of Mathematics, University of Nevada, Las Vegas, Nevada 89154

E-mail address: dwan@nevada.edu 